\title{
Hold off on that shot of Java: more evidence that caffeine intake leads to false negative adenosine stress myocardial perfusion
}

\author{
Tim Leiner ${ }^{1}[$
}

Received: 26 August 2016 / Accepted: 29 August 2016 / Published online: 31 August 2016

(C) Springer Science+Business Media Dordrecht 2016

The presence of coronary atherosclerosis can lead to impaired myocardial perfusion. There is near universal consensus that abnormal myocardial perfusion should guide the decision whether or not invasive treatment is warranted [1]. In clinical practice a variety of diagnostic tests are commonly used to establish impaired myocardial perfusion. Today the most widely used tests are single photon-emission computed tomography (SPECT), positron emission tomography (PET) and magnetic resonance myocardial perfusion imaging (MR-MPI) [2]. An important drawback of these techniques is the need for injection of an exogenous tracer or contrast agent. Apart from the costs associated with these approaches there is also a small but non-negligible risk for acute and chronic side-effects.

A promising alternative technique to assess myocardial perfusion is non contrast-enhanced or 'native' myocardial T1 mapping. Native T1 mapping is a novel MRI technique that can potentially detect myocardial ischemia as well as infarcted myocardium by direct assessment of myocardial water content. $\mathrm{T} 1$ is a magnetic property of tissue that is prolonged by increased water content. In a recent study, Liu et al. found that normal, infarcted, ischemic myocardium can be differentiated by native $\mathrm{T} 1$ mapping at rest and during adenosine stress because these tissues classes exhibit distinctive T1 profiles. Compared to resting conditions, myocardial $\mathrm{T} 1$ values during adenosine stress increased approximately $6 \%$ both at 1.5 and $3.0 \mathrm{~T}$ field strengths in

Tim Leiner

t.leiner@umcutrecht.nl

1 Department of Radiology and Nuclear Medicine, Utrecht University Medical Center, Heidelberglaan 100, 3584CX Utrecht, The Netherlands normal myocardium, whereas in ischemic myocardium the change in T1 was negligible [3]. Although these authors studied a small number of patients and did not report testretest reproducibility data, the study is promising as it potentially allows for safer, more efficient and cheaper MRI examinations.

An important confounder of the response to adenosineinduced myocardial vasodilatation is caffeine. Caffeine (1,3,7-trimethylxanthine) is one of the most widely consumed stimulants worldwide with over $80 \%$ of the US population reporting daily intake [4]. Individuals primarily consume caffeine for a variety of behavioral effects such as changes in mood, energy and alertness [5]. Caffeine is present in coffee, tea, a variety of different soft drinks, energy drinks as well as cacao products and many fruits and vegetables. In the US approximately $75 \%$ of caffeine intake is attributable to coffee consumption with estimated mean intake of approximately $180 \mathrm{mg} /$ day [4]. A number of studies have investigated the effect of caffeine on adenosine stress myocardial perfusion imaging, but there is no consensus on whether inadvertent intake should lead to postponement of a planned examination [6, 7].

Given that native T1-mapping is sensitive to the increase in myocardial water content induced by adenosine administration, one could image that this technique can potentially be used to assess adequacy of vasodilator induced myocardial hyperaemia. In this issue of IJCVI Kuijpers et al. [8] propose that the native $\mathrm{T} 1$ response can be used to assess the adequacy of adenosine-induced stress myocardial hyperperfusion. This would provide a quantitative measure of whether a patient is adequately stressed and would be a significant improvement over currently used methods. In their study, rest and stress T1 mapping was performed in 10 patients who inadvertently drank coffee between 24 and $8 \mathrm{~h}$ prior to the MRI examination, 15 patients who drank 
coffee within $4 \mathrm{~h}$ of the MRI examination and a group of 80 control subjects who reported no caffeine consumption in the $24 \mathrm{~h}$ prior to the MRI examination. Of these 80 patients, 50 patients had normal MRI results, 18 had myocardial ischemia and 12 patients had prior myocardial infarction. Changes in native T1 values were compared as a function of self-reported coffee consumption as well as the presence or absence coronary artery disease. T1 relaxation times were determined in the interventricular septum in every subject as well as in areas with perfusion defects and infarcts. The presence of perfusion defects and infarcts was assessed using contrast-enhanced adenosine stress MRMPI and delayed enhancement imaging. Compared to controls without caffeine intake and normal perfusion, patients who reported caffeine intake in the $24 \mathrm{~h}$ prior to the adenosine stress MR examination were found to have a markedly attenuated T1-response. Whereas native T1 values increased $4.3 \pm 2.8 \%$ in subjects who did not consume caffeine, T1 values decreased by $7.8 \pm 5.0 \%$ in the group of subjects who consumed caffeine $<4 \mathrm{~h}$ before the examination, and increased by just $1.8 \pm 4.0 \%$ in subjects reporting caffeine intake between 8 and $24 \mathrm{~h}$ prior to the examination. There was very little change in T1 value in areas with perfusion defects $(0.2 \pm 0.7 \%)$, and areas with infarcts $(0.3 \pm 0.4 \%)$. Interestingly, in patients who had evidence of ischemia or infarction, remote, visually normal myocardium also exhibited an attenuated $\mathrm{T} 1$ response compared to patients without ischemia or infarction. From these data the authors conclude that the change in myocardial native $\mathrm{T} 1$ value between rest and stress can serve as a measure of stress adequacy and should be reported in the radiology report.

How can these findings be explained? The normal response to adenosine administration is vasodilatation of precapillary coronary arterioles, resulting in an approximately 2.5- to 3.5-fold increase myocardial blood flow [9] in segments subtended by normal coronary arteries. Due to the resultant increase in myocardial blood volume the increase in perfusion leads to measurable changes in $\mathrm{T} 1$ values. Mahmod et al. [10] calculated that the expected increase in $\mathrm{T} 1$ relaxation time is indeed expected to be in the range of 5-6\%, assuming that a threefold increase in flow leads to approximately a doubling of the intramyocardial blood volume, i.e. from an estimated $10 \%$ at rest to $20 \%$ during maximal hyperaemia. When cardiac adenosine receptors are blocked by caffeine, the intended precapillary vasodilation is severely attenuated, which may even lead to a decrease in myocardial blood flow and myocardial blood volume [11], and a reduction in myocardial $\mathrm{T} 1$ values.

In humans, caffeine is metabolized in the liver by the cytochrome P450 (CYP) enzyme CYP1A2 and healthy adults the half-life of caffeine is approximately 4-5 $\mathrm{h}$ [4]. However, there is substantial variation between males and females and different age groups; other important sources of variation between individuals are frequency of caffeine intake, concurrent cigarette smoking and genetic variations in the adenosine receptors and downstream signaling cascade proteins [4]. As demonstrated by Kuijpers et al., native T1-mapping seems capable of detecting the blunting effects of caffeine consumption on adenosine-induced myocardial hyperaemia. Furthermore, the authors postulate that adenosine-induced change in native $\mathrm{T} 1$ values may be a more direct reflection of tissue oxygenation and myocardial ischemia when compared to fractional flow reserve. Indeed, it is well known that a pressure drop across a coronary stenosis does not necessarily result in decreased myocardial perfusion [12], and a more direct measure of downstream tissue perfusion without the need for injection of contrast agents would indeed be hugely valuable.

What do these findings mean for clinical practice? First, this study provides convincing evidence that caffeine consumption indeed influences myocardial perfusion when adenosine is used to induce myocardial hyperperfusion. In light of these findings it seems prudent to emphasize to patients to refrain from caffeine intake in the $24 \mathrm{~h}$ preceding the MRI examination. There are also some caveats that need further attention. Unfortunately, the authors did not repeat stress MR-MPI in all patients who consumed caffeine before the examination. Therefore, we do not know the proportion of patients with false negative MR-MPI results. In addition, blood caffeine levels were not determined and there is no information on test-retest variability in stress and at rest after caffeine consumption. These data are needed before native T1 mapping can be used clinically to assess stress adequacy. It also remains to be investigated whether caffeine blunts native myocardial $\mathrm{T} 1$ reactivity in the presence of other stressors such as the selective adenosine $\mathrm{A}_{2 \mathrm{~A}}$ receptor blocker regadenoson (Lexiscan, Astellas Pharma, Northbrook, IL). A randomized, double-blind, placebo-controlled study of regadenoson-incuded myocardial hyperaemia with ${ }^{15} \mathrm{O}$-labeled water PET by Gaemperli et al. [13] found no significant changes after ingestion of $200 \mathrm{mg}$ caffeine prior to the examination.

In current clinical practice, the 'splenic switch-off' sign is often used as a measure of stress adequacy. The sign-a decrease in splenic contrast-enhancement during concurrent adenosine and gadolinium contrast agent infusion-occurs because adenosine mediates its effects on splenic perfusion via $A_{1}$ and/or $A_{2 B}$ adenosine receptors to maintain circulatory volume in conditions of shock, whereas coronary vasodilatation is mediated via the adenosine $\mathrm{A}_{2 \mathrm{~A}}$ receptor [14]. Although this a very simple and useful way to identify patients at higher risk of a false-negative MR-MPI study, this sign can only be used post hoc, i.e. after contrast has been injected. The attractiveness of the approach by Kuijpers et al. is that it allows adequacy of stress induced hyperperfusion prior to contrast injection, and it provides further 
evidence that native T1 mapping could potentially replace contrast-enhanced MR-MPI.

In conclusion, the study by Kuijpers et al. provides important new insights into the effects of caffeine on myocardial perfusion. In light of the reported results it seems wise to hold off on that shot of java before adenosine stress myocardial perfusion imaging.

\section{Compliance with ethical standards}

Conflict of interest None.

\section{References}

1. Task FORCE Members, Montalescot G, Sechtem U et al (2013) ESC guidelines on the management of stable coronary artery disease: the Task Force on the management of stable coronary artery disease of the European Society of Cardiology. Eur. Heart J 34:2949-3003

2. Takx RAP, Blomberg BA, Aidi El H et al (2015) Coronary artery disease diagnostic accuracy of stress myocardial perfusion imaging compared to invasive coronary angiography with fractional flow reserve. Circ Cardiovasc Imaging 8:e02666. doi:10.1161/ CIRCIMAGING.114.002666

3. Liu A, Wijesurendra RS, Francis JM et al (2016) Adenosine stress and rest $\mathrm{T} 1$ mapping can differentiate between ischemic, infarcted, remote, and normal myocardium without the need for gadolinium contrast agents. J Am Coll Cardiol Cardiovasc Imaging 9:27-36

4. Doepker C, Lieberman HR, Smith AP, Peck JD, El-Sohemy A, Welsh BT (2016) Caffeine: friend or foe? Ann Rev Food Sci Technol 7:117-137

5. Fredholm BB, Bättig K, Holmén J, Nehlig A, Zvartau EE (1999) Actions of caffeine in the brain with special reference to factors that contribute to its widespread use. Pharmacol Rev 51:83-133
6. Saab R, Bajaj NS, Hage FG (2016) Caffeine does not significantly reduce the sensitivity of vasodilator stress myocardial perfusion imaging. J Nucl Cardiol 23:442-446

7. Reyes E (2016) Caffeine reduces the sensitivity of vasodilator MPI for the detection of myocardial ischaemia: pro. J Nucl Cardiol 23:447-453

8. Kuijpers D, Prakken NH, Vliegenthart R, van Dijkman PRM, van der Harst P, Oudkerk M (2016) Caffeine intake inverts the effect of adenosine on myocardial perfusion during stress as measured by T1 mapping. Int J Cardiovasc Imaging. doi:10.1007/ s10554-016-0949-2

9. Vasu S, Bandettini WP, Hsu L-Y et al (2013) Regadenoson and adenosine are equivalent vasodilators and are superior than dipyridamole-a study of first pass quantitative perfusion cardiovascular magnetic resonance. J Cardiovasc Magn Reson BioMed Central 15:85

10. Mahmod M, Piechnik SK, Levelt E et al (2014) Adenosine stress native $\mathrm{T} 1$ mapping in severe aortic stenosis: evidence for a role of the intravascular compartment on myocardial T1 values. J Cardiovasc Magn Reson BioMed Central 16:92

11. Namdar M, Koepfli P, Grathwohl R et al (2006) Caffeine decreases exercise-induced myocardial flow reserve. J Am Coll Cardiol 47:405-410

12. van de Hoef TP, Siebes M, Spaan JAE, Piek JJ (2015) Fundamentals in clinical coronary physiology: why coronary flow is more important than coronary pressure. Eur Heart J 36:ehv235

13. Gaemperli O, Schepis T, Koepfli P et al (2008) Interaction of caffeine with regadenoson-induced hyperemic myocardial blood flow as measured by positron emission tomography: a randomized, double-blind, placebo-controlled crossover trial. J Am Coll Cardiol 51:328-329

14. Manisty C, Ripley DP, Herrey AS et al (2015) Splenic switch-off: a tool to assess stress adequacy in adenosine perfusion cardiac MR imaging. Radiology 276:732-740 